\title{
Endocrine Hypertension
}

\section{Abdelaziz Elamin}

A rterial hypertension a prominent component of a number of endocrine disorders, most prominently those involving the adrenal glands (pheochromocytoma, primary aldosteronism and Cushing syndrome) and the pituitary (ACTH-producing tumors). Hypertension may also be a prominent feature of other endocrine disorders such as acromegaly, thyrotoxicosis, hypothyroidism, and hyperparathyroidism.

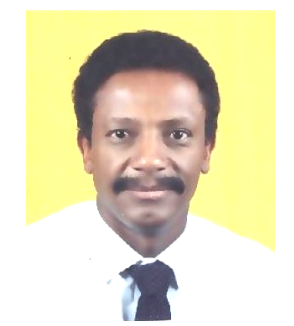
Endocrine hypertension is noted in both adults and children but it is more relevant for children where it comes second to renal hypertension, the common cause of hypertension in that age group.

\section{Hypertension of Adrenal Origin}

The adrenal hormones with greatest effect on blood pressure (BP) are mineralocorticoids. The major adrenal secretory products with mineralocorticoid activity are aldosterone and 11-deoxycorticostereone (DOC). Cortisol also has high intrinsic mineralocorticoid activity but, its actions in the kidney are blunted by local degradation. Aldosterone is produced exclusively in the zona glomeurlosa of the adrenal gland and is primarily controlled by the renin-angiotensin system. Other regulators include sodium $\left(\mathrm{Na}^{+}\right)$and potassium $\left(\mathrm{K}^{+}\right)$plasma concentrations, pituitary $\mathrm{ACTH}$, and dopamine. With the exception of 18-hydroxycorticosterone, the precursors of aldosterone that originate in the zona glomerulosa are normally present in very low concentrations in the peripheral blood. In the zona fasciculata, the two major biosynthetic pathways are under the control of ACTH. The major product formed by the 17hydroxy pathway is cortisol. The principal steroid product of the 17-deoxy pathway with significant mineralocorticoid activity is DOC. Corticosterone and 18hydroxydeoxycorticosterone are also produced in substantial amounts, but these steroids have relatively little mineralocorticoid activity in humans. Aldosterone, in contrast to steroids made in the zona fasciculata, binds weakly to

Professor of Child Health, Endocrine \& Metabolic Diseases

University of Khartoum -Sudan corticosteroid- binding globulin (CBG), and circulates mostly bound to albumin. Free aldosterone comprises $30-50 \%$ of its total plasma concentration, whereas the free fractions of the steroid products of the zona fasciculata comprise $5-10 \%$ of their total concentration. Consequently, aldosterone has a relatively short half-life on the order of 1520 minutes. Aldosterone is rapidly inactivated in the liver, with formation of tetrahydroaldosterone. Another metabolite, aldosterone-18-glucuronide is formed by the kidney and usually represents $5-10 \%$ of the secreted aldosterone, but it has no mineralocorticoid activity. A small amount of free aldosterone appears in the urine and can be easily quantitated. Aldosterone secretion rates vary from 50 to $250 \mu \mathrm{g} /$ day on sodium intake in the range of $100-150 \mathrm{mmol} / \mathrm{day}$. DOC is secreted at approximately the same rate as aldosterone. However, like cortisol. DOC is almost totally bound to CBG, with less than $5 \%$ appearing in the free form. It is metabolized in the liver to tetrahydrodeoxycorticosterone, conjugated with glucuronic acid, and excreted in the urine. There is virtually no free DOC detectable in the urine. The mineralocorticoid activity reflects the availability of free hormone and the affinity of the hormone for the receptor. Aldosterone and DOC have approximately equal and high affinities for the mineralocorticoid receptor and circulate at roughly similar concentrations, but aldosterone is quantitatively the most 
important because much more of it is in the free form. Cortisol has an affinity for the receptor similar to that of aldosterone and its free levels in the circulation are about 100 folds higher than those of aldosterone. Because of this, cortisol is the major steroid that occupies the mineralocorticoid receptors in many tissues such as the pituitary and the heart; however, at normal circulating levels, cortisol does not contribute much to mineralocorticoid action in typical target tissues (kidney, colon, and salivary glands) because of local conversion via $11 \beta-$ hydroxysteroid dehydrogenase to cortisone. Cortisol can lead to mineralocorticoid hypertension when this conversion is blunted by deficiency or inhibition of this enzyme. Aldosterone and other minerlocorticoids influence certain ion-transporting epithelia with high $\mathrm{Na}^{+}-\mathrm{K}^{+}$ATPase levels. The principal effects of the mineralocorticoids are on maintenance of normal $\mathrm{Na}+$ and $\mathrm{K}^{+}$ concentrations and extracellular fluid volume. Mineralocorticoids cross the cell membrane and combine with a mineralocorticoid receptor in the cytosol. The active steroid receptor complex moves into the nucleus of the target cell where it alters the rate of transcription of mineralocorticoid-responsive genes with subsequent change in the levels of specific mRNAs and their protein product. The aldosterone-induced proteins include factors that regulate the luminal $\mathrm{Na}^{+}$channel, facilitating movement of $\mathrm{Na}^{+}$into cells, and components of the $\mathrm{Na}^{+}-\mathrm{K}^{+}$ATPase pump. The principal early effect of aldosterone, beginning in less than 1 hour, is on the $\mathrm{Na}^{+}$ channel. Without altering overall channel abundance, aldosterone increases the apical membrane targeting in the collecting tubules and part of the distal convoluted tubules. Recently a key mediator of this early response was identified as an aldosterone-regulated kinase that increases $\mathrm{Na}^{+}$channel activity. The later effects, 6-24 hours after administration of aldosterone, include activation of $\mathrm{Na}^{+}-\mathrm{K}^{+}$ATPase and alterations in cell morphology and energy metabolism. Some or all of these effects may be due to the altered intracellular $\mathrm{Na}^{+}$concentration, which result from the primary effect on the $\mathrm{Na}^{+}$ channel. In addition to directly enhancing $\mathrm{Na}^{+}$ absorption, the major effect of these changes in ion transport is to increase the difference in electrical potential across the renal tubule. The increased luminal negativity augments tubular secretion of $\mathrm{K}^{+}$by the principal cell and hydrogen ions $\left(\mathrm{H}^{+}\right)$by the intercalated cell. Tubular $\mathrm{Na}^{+}$enters the extracellular fluid via the $\mathrm{Na}^{+}$pump and helps maintain its normal composition and volume. All of these events occur in other secretory systems as well and can be measured in saliva, sweat, and stool.

\section{PATHOGENESISOF MINERALOCORT- ICOID HYPERTENSION}

Mineralocorticoid hormones produce hypertension by several mechanisms. The initiating events are the physiologic consequences of mineralocorticoid-induced expansion of plasma and extracellular fluid volume. The understanding of these mechanisms comes form studies in normal subjects given high doses of mineralocorticoids and form sequential observations made following discontinuation of spironolactone therapy in patients with aldosterone-secreting adenomas. Initially, $\mathrm{Na}^{+}$ and fluid retention occur, with an increase in body weight, extracellular fluid volume, and cardiac output. After gaining 1-2 liters of additional extracellular fluid, the phenomenon of $\mathrm{Na}^{+}$"escape" follows so that a new steady state is achieved. Renal $\mathrm{K}^{+}$wasting persists and arterial blood pressure continues to increase. Typically, the chronic stage of mineralocorticoid excess is characterized by an increase in total peripheral vascular resistance and normalization of stroke volume and cardiac output. The increase in peripheral vascular resistance is related in part to increased sensitivity to catecholamines even without a distinct increment in plasma adrenaline or noradrenaline levels. An additional mechanism may be a direct central action of aldosterone. Infusion of aldosterone in the ventricles of the brains of rats produced hypertension that could not be reversed or prevented by infusion, at the same site, of a 
competitive aldosterone antagonist. Primary mineralocorticoid excess is manifested by hypertension, hypokalemia, and suppression of the renin-angiotensin system. Clinically similar syndromes can result from increased adrenal production of other steroids with mineralocorticoid activity like DOC, failure to inactivate cortisol (the syndrome of apparent mineralocorticoid excess), or as a consequence of mineralocorticoidindependent augmentation of renal $\mathrm{Na}^{+}$ reabsorption due to a constitutively activated epithelial $\mathrm{Na}^{+}$channel (e.g. Liddle's syndrome ). The presence of these latter disorders is often suggested by clinical findings of mineralocorticoid excess in a patient with subnormal aldosterone levels.

\section{Primary Hyperaldosteronism:}

Increase production of aldosterone by abnormal zona glomerulosa tissue (adenoma or hyperplasia) initiates the series of events that result in the typical clinical manifestations of the syndrome of primary hyperaldosteronism. A benign aldosteroneproducing adenoma, as originally described by Conn, accounts for $75 \%$ of cases of primary hyperaldosteronism. Idiopathic hyperaldosteronism, a disorder with many similar clinical features, accounts for most of the remaining cases. In idiopathic hyperaldosteronism the adrenals are either normal in appearance or, more commonly, reveal bilateral micro or macronodular adrenal hyperplasia. Increased renal $\mathrm{Na}^{+}$ retention results in expansion of the extracellular fluid volume and increased total body $\mathrm{Na}^{+}$content. Although the effects on the kidney are greatest quantitatively, other mineralocorticoid target tissues are also affected. Fecal excretion of $\mathrm{Na}^{+}$, for example, can be decreased to almost nil, with a measurable effect on the rectal potential difference. Salivary electrolyte ratios can also reflect the influence of hyperaldosteronism on that target tissue. The expanded extracellular fluid and plasma volumes are sensed by stretch receptors at the juxtaglomerular apparatus and $\mathrm{Na}^{+}$flux at the macula densa, with resultant suppression of renin secretion, measured as suppressed plasma renin activity. Suppression of the renin-angiotensin system, while not of it self diagnostic of primary aldosteronism, is thus a major feature of this disorder. In addition to $\mathrm{Na}^{+}$retention potassium depletion develops, decreasing the total body and plasma concentration of $\mathrm{K}^{+}$. The extrusion of potassium from its intracellular reservoir is followed by the intracellular movement of $\mathrm{H}^{+}$and, together with aldosterone-dependent increase in renal secretion of $\mathrm{H}^{+}$, results in metabolic alkalosis. With moderate potassium depletion, decreased carbohydrate tolerance and increased resistance to vasopressin occur leading to polyuria. Severe $\mathrm{K}^{+}$depletion blunts baroreceptor function, occasionally producing postural hypotension. Primary hyperaldosteronism is a disease of the zona glomerulosa. Other adrenal product formed in this zone such as DOC, corticosterone, and 18-hydroxycorticosterne may be present in increased amounts in the blood or urine of persons with an aldosterone producing adenoma. Cells of this zone do not have the ability to make cortisol. Thus, there are no abnormalities in either cortisol production or metabolism. Plasma and urine cortisol levels are normal.

Clinical Features: Patients typically come to medical attention because of symptoms of hypokalemia or detection of previously unsuspected hypertension during the course of a routine physical examination. The medical history reveals no characteristic symptoms other than non specific complaints of fatigue, loss of stamina, weakness, and lassitude, all of which are symptoms of $\mathrm{K}^{+}$depletion. If $\mathrm{K}^{+}$ depletion is more severe, thirst, polyuria, headache and paresthesias may also be present. Excessive production of mineralocorticoids produces no characteristic physical findings. Blood pressure in patients with primary hyperaldosteronism can range from borderline elevation to severe hypertension, but accelerated or malignant hypertension is extremely rare. Orthostatic decreases in blood pressure without reflex tachycardia are observed in the severely $\mathrm{K}^{+}$ depleted patient because of blunting of the 
baroreceptors signals. A positive Trousseau or Chvostek sign is suggestive of alkalosis and hypocalcemia that accompany severe potassium depletion. The heart is usually only mildly enlarged and electrocardiographic changes reflecting modest left ventricular hypertrophy and potassium depletion may be detected. Frank heart failure and clinical edema are uncommon.

Diagnosis: Detection of spontaneous hypokalemia is often the initial clue that suggests a diagnosis of primary hyperaldosteronism in a patient with hypertension. During the investigation, a high potassium diet or potassium supplements should be avoided, and all previous diuretic therapy must be discontinued for at least three weeks before a valid serum or plasma potassium measurement can be obtained. The most common cause of hypokalemia in patients with hypertension is diuretic therapy. In some series, up to $20 \%$ of patients with primary hyperaldosteronism have had normal serum potassium concentrations. Serum potassium concentration is closely related to and determined to a great extent by sodium intake. A low $\mathrm{Na}^{+}$diet, by reducing delivery of $\mathrm{Na}^{+}$to aldosterone-sensitive sites in the distal nephron, can reduce renal $\mathrm{K}^{+}$secretion and thus correct hypokalemia. On the other hand, increased distal delivery of $\mathrm{Na}^{+}$ accompanying a high $\mathrm{Na}^{+}$diet can enhance $\mathrm{K}^{+}$ loss, particularly when aldosterone is being secreted autonomously and is therefore not subject to the normal suppression by the high $\mathrm{Na}^{+}$intake. These physiologic relationships serve to illustrate the importance of controlling the dietary $\mathrm{Na}^{+}$intake when evaluating patients suspected of having primary hyperaldosteronism. In the presence of normal renal function and autonomous aldosterone production, salt loading will usually unmask hypokalemia. In most of the western countries, the average person consumes more than $120 \mathrm{mmol}$ of $\mathrm{Na}^{+}$per day, enough to allow hypokalemia to become manifest when present. If a dietary history of high salt intake is obtained and the $\mathrm{K}^{+}$ concentration is normal, a diagnosis of primary hyperaldosteronism is unlikely.
Patients who report a low $\mathrm{Na}^{+}$intake should be advised to take an unrestricted diet plus $1 \mathrm{~g}$ of sodium chloride with each meal for four days and blood samples for electrolyte determinations should be obtained in the fasting state on the following morning. This dietary regimen is also useful because it prepares the patient for optimal measurement of renin and aldosterone levels. Assessment of the renin-angiotensin system can be accomplished by a random plasma rennin activity measurement. If plasma rennin activity is normal or high in a patient who has not been receiving diuretic therapy for at least three weeks, it is very unlikely that an aldosterone-producing adenoma is present. However, some patients with idiopathic hyperaldosteronism may have low normal levels of plasma rennin activity. On the other hand, a subnormal plasma renin level is not alone sufficient to establish a diagnosis of primary hyperaldosteronism, since a large subgroup of patients with essential hypertension have low plasma renin levels. If hypokalemia and suppressed renin activity are detected, plasma and urinary aldosterone measurements should be obtained while the patient is taking an unrestricted salt diet. This is crucial, because with any significant diminution of slat intake, plasma aldosterone concentration and aldosterone production normally increase. Assessment of aldosterone production can best be accomplished by measurement of urinary excretion of aldosterone or one of its metabolite over a 24 hour period. Most laboratories measure excretion of the 18-glucuronide metabolite. The normal rate of urinary excretion of this metabolite ranges from $5-20 \mu \mathrm{g} /$ day. Urinary measurements are superior to random measurements of plasma aldosterone for the detection of abnormal production of aldosterone but are not always able to discriminate between patients with adenoma and those with idiopathic hyperaldosteronism. Samples for measurements of plasma aldosterone concentration should ideally be obtained at around 8:00 AM after at least 4 hours of recumbency and under the same dietary conditions as described above for 
measurement of urinary aldosterone. This measurement not only confirms the presence of hyperaldosteronism but also provides insight into the probable underlying pathology. When obtained under these conditions, a plasma aldosterone concentration greater than $25 \mathrm{ng} / \mathrm{dl}$ (695pmol/1) usually indicates the presence of an aldosterone-producing adenoma. The plasma aldosterone level should also be determined after 2-4 hours in the upright posture, which normally activates the rennin system with a resultant increase in the plasma aldosterone level. Ninety Percent of patients with adenoma will show no significant change or an obvious decrease in plasma aldosterone levels, whereas aldosterone levels almost always increase in those with idiopathic hyperaldosteronism. The difference is due to: (1) The profound suppression of the renin system by excessive aldosterone production in patients with an adenoma (2) the influence of ACTH, to which the adenoma is still responsive and which normally decreases between $8 \mathrm{AM}$ and 12 noon; and (3) decreased responsiveness of adenomas to angiotensin-II. In contract in patient with idiopathic hyperaldosteronism, increased sensitivity of the gland to small increase in renin and angiotensin-II levels that occur in the upright posture leads to an increased aldosterone secretion. Serum cortisol levels must be measured simultaneously. An increase in serum cortisol implies an ACTH discharge and invalidates the information obtained from the maneuver. Some investigators have advocated the use of the aldosterone/renin ratio as a means of screening for and perhaps establishing a diagnosis of primary hyperaldosteronism. A ratio in excess of 30 (assuming that aldosterone levels are reported in nanograms per deciliter and renin levels in nanograms per milliliter per hour) is considered abnormal. However, since a low renin level (e.g. 0.1 $\mathrm{ng} / \mathrm{ml} / \mathrm{h}$ ) can result in an elevated ration even when aldosterone levels are in the low normal range (e.g. $3 \mathrm{ng} / \mathrm{dl}$ ), use of the ratio in the absence of a concomitantly elevated aldosterone level should be discouraged. Measurement of other adrenal steroids may add to the precision of diagnosis. Plasma DOC, corticosterone, and particularly, 18hydroxycorticosterne levels are frequently increased in patients with primary hyperaldosteronism, whereas they are rarely if ever increased in patients with idiopathic hyperaldosteronism. Increased urinary excretion of 18-hydroxycrotisol and 18oxocortisol, which is a characteristic finding in individuals with the dexamethasonesuppressible form of primary hyperaldosteronism, may also be present in some patients with aldosterone-producing adenomas. The procedures just discussed can confirm a diagnosis of primary hyperaldosteronism and differentiate adenoma from idiopathic hyperaldosteronism in most patients. Localization studies can also provide additional information. When uncertainty persists, the saline infusion test may be useful. Saline loading establishes aldosterone unresponsiveness to volume expansion and thereby identifies autonomy in patients with aldosterone-producing adenomas. Two litres of isotonic saline are administered over 24 hours. Blood samples for aldosterone and cortisol measurements are obtained before and after the infusion. Expansion of the extracellular fluid volume reduces plasma, aldosterone concentration promptly in patients with idiopathic hypertension but fails to suppress plasma aldosterone concentration into the normal range in patients with adenoma or hyperplasia. Moreover, the saline infusion test typically distinguishes patients with primary hyperaldosteronism from those with low-renin essential hypertension. The ratio of aldosterone to cortisol is typically greater than 3.0 following administration of saline in patients with an aldosteroneproducing adenoma reflecting the limited effect of volume expansion in the setting of marked suppression of the renin-angiotensin system. 


\section{Rare Form of Primary Hyperaldo stero- nism}

A:Dexamethasone-Remediable Aldosteronism: This is a rare form of genetic hypertension that has been recognized with increasing frequency since the molecular basis of this disorder was established. It is inherited in an autosomal dominant pattern. The primary defect is a gene duplication that results from an unequal crossing over event that fuses the regulatory region of the $11 \beta$ hydroxylase gene to the coding sequence of aldosterone synthase. With cortisol as a substrate, expression of this enzyme in the zone fasciculata results in the synthesis of 18hydroxycortisol and 18-oxocortisol (phenotypic markers of this disorder). Aldosterone secretion also occurs in this zone and is ACTH-dependent, such that small doses of dexamethasone (1-2 mg/day) can ameliorate hypertension as well as the typical biochemical findings. However, the response to dexamethasone suppression may diminish with prolonged use in many patients, and additional antihypertensive therapy may thus be necessary. The presence of dexamethasone-remediable hypertension should be considered in any family with primary hyperaldosteronism. The diagnosis can easily be established by measurement of the marker steroids (18-oxocortisol \&18hydroxycortisol) or by detection of the abnormal gene in the DNA samples.

\section{B:Aldosterone-producing} carcinoma:

Malignant adrenal tumors producing aldosterone in the absence of hypercortisolism is rare, accounting for less than 3\% of cases of primary hyperaldosteronism. In general, the biochemical and hormonal features and the response to dynamic tests are similar to those in patients with adenoma except that the size of the anomalies is greater. Hypercortisolism, hyperandrogenism and hyperestrogenism may also be found.

\section{Management of Aldosterone-producing Adenomas:}

Once the biochemical diagnosis is secure, localization of the site of the adenoma helps with surgical management. A number of techniques are in use including: CT and MR imaging, adrenal isotope scanning and adrenal vein catheterization. The diagnostic information provided by $\mathrm{CT}$ and MRI in locating adenomas has proved to be both accurate and practical and thus it is the initial procedure of choice. Scanning using radioisotopes can identify the tumor in $80 \%$ of patients, but success rate decreases markedly if the tumor is less than $1 \mathrm{~cm}$, in diameter. Obtaining blood samples from adrenal veins on both sides for measurement and comparison of aldosterone level continues to be useful in lateralizing tumors after other techniques fail. Cortisol levels should be measured simultaneously to confirm the source of the sample and extent of mixing with non-adrenal blood. Injection of dye in the adrenal vein (venography) is hazardous and is no longer utilized. Treatment depends on accurate diagnosis. When the diagnosis and lateralization is certain, surgical removal of the adenoma or unilateral adrenalectomy via a laparoscope is recommended. Ideally, patients should be treated preoperatively with spironolactone until the $\mathrm{BP}$ and serum $\mathrm{K}^{+}$are normal. This drug is particularly beneficial because it blocks the mineralocorticoid receptor and inhibits aldosterone synthesis by adenomas. It normalizes the extracellular fluid volume, promotes $\mathrm{K}^{+}$retention and activates the suppressed renin-angiotensin system, which in turn promotes normal secretion of aldosterone by the healthy gland. Postoperative hypoaldosteronism and hyperkalemia is unlikely with this kind of action. Spironolactone is well tolerated usually; the side effects of rashes, gynecomastia, impotence and epigastric discomfort are rare and disappear with continuation of medication. In patients who fail to tolerate the drug, the $\mathrm{K}^{+}$-sparing diuretic amiloride can be used as an alternative. Other antihypertensive drugs may also be required to obtain optimal control of BP. Calcium channel blockers are particularly effective in this setting. The postoperative cure rate of hypertension is recorded in over $70 \%$ of patients in most studies, with partial 
reduction of hypertension in the remainder. Subtotal adrenalectomy will correct hypokalemia in patients with idiopathic hyperaldosteronism but hypertension is rarely cured. Therefore, antihypertensive medication is needed and such patients should not be routinely sent to surgery. A subset of patients with primary hyperaldosteronism but no identifiable adenoma, referred to as primary adrenal hyperplasia, also benefit from surgical reduction of adrenal mass. If surgery is contraindicated or refused by the patient, prolonged treatment with spironolactone can be effective. The initial dose of 200-400 $\mathrm{mg} /$ day must be continued for 4-6 weeks before the full effect on BP is realized, but a maintenance dose of $75-100 \mathrm{mg} /$ day is usually sufficient to keep the BP under control.

\section{DEOXYCORTICOSTERONE EXCESS (DOC)}

DOC is the second most important naturally occurring mineralocorticoid hormone. Accordingly, excess DOC should be suspected in any hypertensive patient with hypokalemia and suppression of renin and aldosterone production.

\section{CONGENITAL ADRENAL HYPER- PLASIA}

11ß-hydroxylase Deficiency: Congenital adrenal hyperplasia due to $11 \beta$-hydroxylase deficiency is usually recognized in newborns and infants because of virilization and the presence of hypertension and hypokalemia. Plasma androgens, 11-deoxycortisol, 17 $\alpha$ hydroxyprogesterone, urinary 17-ketosteroids, and 17-hydroxycorticosteroid are increased. The defect has been identified in the gene, which is mapped on chromosome 8. It is usually partial, so that some cortisol is produced but it does not increase with further ACTH stimulation. Blood levels of cortisol are usually within normal limits. A partial defect of 11ß-hydroxylation results in increased production and high blood levels of DOC, 11-deoxycortisol, and androgens. Hypertension results from excessive production of DOC by mechanisms similar to those described for aldosterone over secretion earlier. The blood levels and production rates of aldosterone are normal or reduced in this condition. Two mechanisms are proposed. Originally, a partial deficiency of the $11 \beta$ hydroxylation activity in the zona glomerulosa was postulated, with a block in aldosterone synthesis. This concept was supported by the observation that after normalization of DOC production and correction of the hypertension by $\mathrm{ACTH}$ suppression, aldosterone production remained normal or reduced and a $\mathrm{Na}^{+}$-losing state could be provoked. Currently, it is felt that there is no defect in the zona glomerulosa but that suppression of renin by the increased production of DOC reduces the production of aldosterone. Thus, after chronic salt restriction and ACTH suppression, both renin and aldosterone dynamics return to normal, implying an intact zona glomerulosa.

17a-Hydroxylase Deficiency: The deficiency of this key enzyme in the steroid biosynthesis cycle prevents production of androgens and estrogens, and decreases production of cortisol. The defect occurs in a single gene on chromosome 10, which codes for the enzyme. The diminution in cortisol production induces surge in ACTH production. As a result all non 17-hydroxylated steroids products are increased including DOC and aldosterone. The subsequent expansion of extracellular fluid and blood volume leads to hypertension and suppression of the renin-angiotensin system, which in turn causes suppression of aldosterone production. Thus the principal steroids present in excess are: DOC, corticosterone, 18-hydroxycortisol and 18hydroxydeoxycorticosterone. There has been no instance in which the adrenal defect occurs without the gonadal defect and the condition is usually recognized at the time of puberty by presence of hypertension, hypokalemia and primary amenorrhea in the female or feminized genitalia in the male. Patients often have eunuchoid proportions and younger age appearance. The virtual absence of $17 \alpha$ hydroxyprogestrone, pregnanetriol, and 17 keto-steroids is diagnostic. 
Treatment of $17 \alpha$ and $11 \beta$-Hydroxylase

Deficiency: Treatment of both of these disorders is similar to that of all non- $\mathrm{Na}^{+}-$ losing forms of congenital adrenal hyperplasia. Treatment with physiologic replacement doses of glucocorticoid, such as hydrocortisone or dexamethasone, restores blood pressure to normal levels, corrects $\mathrm{K}^{+}$ depletion, reduces excessive DOC and corticosterone production in $17 \alpha$-hydroxylase deficiency, and reduces DOC and 11deoxycortisol production in $11 \beta$-hydroxylase deficiency. In $17 \alpha$-hydroxylase deficiency syndrome, restoration of normal levels of DOC results in a return of plasma renin activity and aldosterone to normal values. A delay in return of the suppressed reninaldosterone system toward normal can result in hypovolemic crises with initial natriuresis and diuresis. It may take several years before the aldosterone, and renin systems become normal. The amount of glucocorticoid administered must be carefully determined because of apparently exquisite tissue sensitivity to glucocorticoid hormones. Addition of estrogen-progesterone combined cyclic therapy may be necessary in the adult patient with $17 \alpha$-hydroxylase deficiency.

\section{Androgen \& Estrogen-Producing Adrenal}

Tumors: Most of the C-19 steroids produced by the adrenocortical zona reticularis have weak androgen activity, especially dehydroepiandrosterone (DHEA) and it is sulfate (DHEAS) as well as androstenedione. Disturbance in both internal zona reticularis regulatory mechanisms (enzyme activity) and its extra-adrenal regulators (ACTH and an androgen-stimulating peptide of possible hypothalamic-pituitary origin) may lead to excessive adrenal sex steroid production, resulting in syndromes of hirsutism and virilization in females or feminization in males. Although the zona reticularis has no intrinsic capacity to synthesize any effective glucocorticoid or mineralocorticoid, it has the potential, under conditions of chronic stimulation by $\mathrm{ACTH}$, to transform some cellular function into the fasciculata cell type and produce cortisol and presumably other typical zona fasciculata steroids. Some patients with malignancies originating in the zona reticularis may have clinical features of mineralocorticoid excess, with hypertension, hypokalemia, and renin suppression. Aldosterone levels are generally not elevated and are often reduced. Urinary or plasma steroid profiling in some of these patients suggests that there may be inhibition of $11 \beta$ hydroxylase activity in association with the increased production of androgens or estrogens. Administration of methylandrostanediol to experimental animals and testosterone to humans suggests that exogenous androgen excess can block the conversion of 11-deoxycortisol and DOC to cortisol and corticosterone, respectively. Excessive secretion of DOC could then lead to a state of mineralocorticoid excess. Increased urinary excretion of DOC metabolites or plasma DOC concentrations have been found in several patients with androgen or estrogen-producing adrenocortical carcinomas who have hypertension and hypokalemia. The inhibition of $11 \beta$-hydroxylase in these patients may be due to inactivation of the enzyme cytochrome CYP11B1 by the high intra-adrenal concentration of androgens that act as a pseudosubstrate for the reaction. This mechanism is similar to that occurring in Cushing's syndrome, in which cortisol appears to serve as the enzymatic inhibitor.

\section{Syndrome of Primary Cortisol Resistance:} Peripheral resistance to cortisol action is a very rare condition in which severe hypertension, hypokalemia, and renin suppression are associated with elevated plasma and urinary levels of cortisol without causing clinical manifestations of Cushing's syndrome. The basic defect is at the level of the glucocorticoid receptor; both the number of receptors and the affinity of the receptors for cortisol are reduced in the target tissues. In this condition, plasma levels of ACTH are elevated as a result of block of cortisol feedback at the corticotroph. Cortisol production is thus increased, but it does not result in the typical clinical stigmas of 
hypercortisolism. However, chronic stimulation by excess ACTH of the 17-deoxy pathway of the zona fasciculata results in abnormal production of DOC and corticosterone, causing hypertension, hypokalemia, and suppression of renin and aldosterone production. The clinical and biochemical abnormalities are partially relieved by treatment with high doses of dexamethasone.

\section{CUSHING'S SYNDROME}

Hypertension is a common finding of endogenous hypercortisolism but occurs in only $10-20 \%$ of patients receiving therapy with synthetic glucocorticoids. ACTHdependent hypercortisolism (Cushing's disease and ectopic ACTH production) is frequently accompanied by increased levels of other ACTH-dependent steroids, especially DOC and corticosterone. The elevated DOC and cortisol levels probably contribute to the mineralocorticoid excess state. Plasma renin activity varies but is typically normal as a consequence of concomitant increase in the production of angiotensinogen. Serum $\mathrm{K}^{+}$ levels are also normal in most patients, implying the absence of a mineralocorticoid excess state. However, a small subset of patients has hypokalemia and suppressed plasma renin levels. Most of these patients have ectopic ACTH hypersecretion or adrenal tumors. Even when there is evidence for mineralocorticoid excess, suppressed plasma renin and hypokalemia, levels of aldosterone and 18-hydroxycorticosterone are consistently within or below the normal range. Hypertension in Cushing's syndrome is usually more frequent in patients with adrenocortical hyperplasia, due to ACTH excess, than in patients with cortical adenomas, which implies that, in addition to cortisol, other ACTH-dependent steroids (DOC, corticosterone \& 18hydroxydeoxycorticosterone) may contribute to the development or maintenance of hypertension. In addition, urinary excretion of the potent naturally occurring mineralocorticoid 19-nor-DOC, produced in the kidney by the conversion of an oxygenated form of DOC, is elevated in both the primary (adrenal) and secondary (pituitary) forms of Cushing's syndrome. Since most patients with Cushing's syndrome do not have findings consistent with hyperminealocorticoidism, glucocorticoids appear to cause hypertension by mineralocorticoid-independent mechanisms. These include increased production of angiotensin-II due to glucocorticoid-induced increases in the hepatic synthesis of angiotensinogen; enhanced glucocorticoidmediated vascular reactivity to vasoconstrictors; inhibition of extra neuronal uptake and degradation of catecholamines; inhibition of vasodilatory systems such as kinins and prostaglandins; shift in $\mathrm{Na}^{+}$from the intracellular to the extracellular compartment, resulting in increased plasma volume; and an increase in cardiac output from the increased production of adrenaline due to enhanced phenylethanolamine-Nmethyltransferase activity in the adrenal medulla.

\section{PSEUDOHYPERALDOSTERONISM:}

The term pseudohyperaldosteronism comprises heterogeneous group of disorders in which the clinical features are consistent with mineralocorticoid excess yet endogenous mineralocorticoid secretion is abnormally low due to suppressed renin production. Renin secretion is suppressed by increased $\mathrm{Na}^{+}$ retention and volume expansion, resulting either from the presence of endogenous or exogenous mineralocorticoids or mineralocorticoid-like substances or from a mineralocorticoid-independent increase in renal tubular sodium transport. Hypertension, hypokalemia, and metabolic alkalosis are the usual manifestations. In addition to the syndrome that result from excess production of DOC as described above, continuous use of fluorinated steroids with powerful mineralocorticoid-like activity contained in some topical preparation such as nasal sprays and dermatologic creams can be associated with hypertension, hypokalemia and renin and aldosterone suppression. Withdrawal of these medications or adjustment of the dosage 
easily controls undesirable side effects. Pseudohyperaldosteronism can also occur as a prominent feature of several rare syndromes, the pathophysiologic features of which have only recently been established.

\section{Syndrome of Apparent Mineralocorticoid}

Excess: A rare disorder, initially designated as the syndrome of apparent mineralocorticoid excess, is characterized by findings suggestive of a hypermineralocorticoid state: hypertension, hypokalemia, suppressed renin levels, and amelioration by spironolactone, despite low levels of aldosterone and DOC. Most of the reported cases have been in children who have severe hypertension. Although the pathogenesis remained elusive for more than a decade, it is now evident that the primary abnormality in this disorder is reduced peripheral metabolism of cortisol due to a mutation in the gene encoding $11 \beta$ hydroxysteroid dehydrogenase type 2 , the isoenzyme that is present in greatest abundance in the renal tubule. Impaired conversion of cortisol to cortisone in the cells of the renal tubule results in an accumulation of cortisol and subsequent occupancy of mineralocorticoid receptors. Despite normal plasma cortisol levels, urinary cortisol is increased, reflecting impairment of the kidney's ability to convert cortisol to cortisone. The increased urinary excretion of tetrahydrocortisol (THF) and reduced excretion of tetrahydrocortisone (THE) leads to a marked increase in the THF/THE ratio, a finding considered diagnostic of this disorder. Treatment consists of the administration of small doses of dexamethasone $(0.75-1 \mathrm{mg} / \mathrm{d})$ to suppress ACTH and to limit thereby the production of cortisol and its accumulation in mineralocorticoid target tissues in the kidney.

Chronic Ingestion of Licorice: Chronic ingestion of large amounts of substances containing mineralocorticoid-like activity, e.g. certain candies, infusions, drinks and some chewing tobaccos containing licorice, results in a syndrome of hypertension, hypokalemia, renal $\mathrm{Na}^{+}$retention, volume expansion, suppressed plasma renin activity, and metabolic alkalosis. The secretion and excretion of aldosterone and its precursors are low or undetectable. The responsible agent for this syndrome is the active principle of licorice, glycyrrhizic acid, and its metabolite glycyrrhetinic acid, that are present in certain commercially available products. Both of these alkaloids inhibit $11 \beta$-hydroxysteroid dehydrogenase in the kidney, which increases free cortisol locally to act as the mineralocorticoid in a manner similar to the syndrome of apparent mineralocorticoid excess. Pseudohyperaldosteronism can be induced by licorice and its derivative carbenoxolone, which is used in treatment of gastric ulcers. Electrolyte abnormalities and hypertension disappear within a few weeks upon discontinuation of licorice ingestion or withdrawal of carbenoxolone treatment.

Liddle's Syndrome: In 1963, Liddle and his colleagues reported the results of studies in a large family in which the affected members had clinical manifestations resembling those of primary hyperaldosteronism. Aldosterone production, however, was negligible. The inheritance pattern in this family was that of an autosomal dominant disorder, and the phenotype was soon identified in several additional families as well as in sporadic cases. Although these findings suggest the possibility of excessive production of another mineralocorticoid, none has been documented. In contrast to patients with excess DOC production or those with the syndrome of apparent mineralocorticoid excess, administration of the mineralocorticoid antagonist spironolactone to patients with Liddle's syndrome did not correct neither the hypertension, nor the hypokalemia. Furthermore, the glucocorticoid synthesis blocking agent metyrapone, which inhibits $11 \quad \beta$ and 18-hydroxylation of aldosterone precursors, also had no effect. Interestingly, administration of triamterene, a diuretic agent with $\mathrm{K}^{+}$sparing activity independent of mineralocorticoid antagonism, was effective in controlling the hypertension and the hypokalemia. The investigators 
proposed that a primary defect in the renal tubule, which enhances $\mathrm{Na}^{+}$reabsorption, was responsible. Recent studies on many families and patients with this syndrome have supported the suggested hypothesis. Using linkage studies as well as electrophysiologic techniques, it has been established that patients with Liddle's syndrome have a defect in the cytoplasmic domain of either the $\beta$ or $\gamma$ subunit of the epithelial $\mathrm{Na}^{+}$channel that result in constitutive activation of the channel. Since triamterene as well as amiloride are relatively specific inhibitors of this channel, treatment with these agents will correct the hypokalemia and ameliorate the hypertension.

Type II Pseudohypoaldosteronism: The term type II pseudohypoaldosteronism, also known as Arnold-Healy-Gordon Syndrome, has been used to describe a rare clinical syndrome in which hypertension is present in association of hyperkalemia, impairment of renal $\mathrm{K}^{+}$excretion, hyperchloremic metabolic acidosis and hyporeninemic hypoaldosteronism. The GFR is usually normal and the mineralocorticoid resistance is shown by persistence of hyperkalemia and subnormal kaliuretic response to large doses of exogenously administered mineralocorticoid hormone. However, in contrast to patients with classic form of mineralocorticoid resistance (type 1 pseudohypoaldosteronism), salt wasting is not present and both the anti-natriuretic and antichloruretic responses to mineralocorticoid are intact. Impaired renal $\mathrm{K}^{+}$secretion was initially proposed as the primary defect. However, whereas fractional renal $\mathrm{K}^{+}$ excretion was subnormal and increased only minimally when $\mathrm{Na}^{+}$was delivered to the distal nephron segments as $\mathrm{NaCl}$, distal renal $\mathrm{K}^{+}$secretion increased greatly when $\mathrm{Na}^{+}$was delivered distally in the presence of non- $\mathrm{Cl}^{-}-$ anions (sulfate and bicarbonate). These findings indicate that the renal $\mathrm{K}^{+}$secretory mechanism is intact and suggested an alternative hypothesis in which the primary defect was proposed to increase the reabsorptive avidity of the distal nephron for $\mathrm{Cl}^{-}$. This, in turn, would (1) limit the $\mathrm{Na}^{+}$and mineralocorticoid-dependent driving force for $\mathrm{K}^{+}$and $\mathrm{H}^{+}$secretion, resulting in hyperkalemia and acidosis; and (2) augment distal $\mathrm{NaCl}$ reabsorb ion, resulting in hyperchloremia, volume expansion, and hypertension. Consistent with the presence of such a "chloride shunt" restriction of dietary $\mathrm{NaCl}$ or administration of a chloruretic diuretic (furosemide, thiazides) ameliorates hyperkalemia and acidosis in such patients.

\section{OTHER HORMONE SYSTEMS \& HYPERTENSION}

INSULIN: Hyperinsulinemia and insulin resistance have been implicated as potential factors in the generation of hypertension, particularly in obese patients. It has been argued that insulin resistance is present in most obese patients with hypertension and in some non-obese hypertensive patients. In the setting of obesity there is impaired insulinmediated glucose uptake resulting in both type 2 diabetes mellitus and increased insulin secretion by the pancreas (hyperinsulinemia). The distribution of body fat may also be a key factor, in as much as hypertension and insulin resistance are seen more commonly in patients with abdominal obesity. The association of hypertension, diabetes mellitus, abdominal obesity, and hyperlipidemia has been referred to as "syndrome $X$ " or the syndrome of insulin resistance. The hypertension observed in this clinical syndrome may be due in part to the hyperinsulinemia. Insulin accentuates the activity of the sympathetic nervous system, leading to greater vasoconstriction. In addition, insulin increases renal $\mathrm{Na}^{+}$ reabsorption, resulting in increased intravascular volume and blood pressure. Although insulin usually induces vasodilatation to counter-balance these pressor forces, in the setting of obesity this action is attenuated. Thus, in these insulinresistant states, it is postulated that the stimulation of both the sympathetic nervous system and renal $\mathrm{Na}^{+}$reabsorption by hyperinsulinemia combined with impaired vasodilatation results in increased blood pressure. It is noteworthy that weight loss 
lowers blood pressure, insulin levels, and insulin resistance in these patients. Increase insulin levels alone are probably not sufficient to cause hypertension given the observations that experimental animals receiving high doses of insulin and patients with insulinomas do not develop hypertension. In addition, there are a substantial number of patients with obesity, insulin resistance, and type 2 diabetes mellitus who do not have hypertension (e.g. Pima Indians in the US). Thus, there is probably a critical interplay of genetic and hormonal factors in the pathogenesis of hypertension in patients with insulin resistance.

NATRIURETIC PEPTIDES: Extracts of aterial but not ventricular tissue cause marked natriuresis when injected into rats. The material is contained in densely staining granules in the atria of most mammalian species. Atrial natriuretic peptide (ANP) is a 28-amino-acid peptide derived from the cleavage of the carboxyl terminal of a 126amino-acid precursor located primarily in the storage vesicles of atrial cells. At least three other natriuretic peptides have subsequently been identified; a brain natriuretic peptide (BNP) C-type peptide (CNP), and a renal natriuretic peptide (urodilatin). Although originally described in the brain, the major source of BNP is the cardiac ventricle, and its action is similar to that of ANP. CNP is mainly produced in the brain, where it serves as a neurotransmitter and in endothelial cells, where it may regulate vasoconstriction. Urodilatin is produced in the kidney, where it acts locally to affect $\mathrm{Na}^{+}$transport. These natriuretic peptides bind to membrane receptors linked to guanylyl cyclase, resulting in production of the second messenger, cGMP. The major effects seen following the administration of ANP are vasodilatation, hyperfiltration, and natriuresis. Although the peptide can cause relaxation of vascular smooth muscle the fall in blood pressure is thought to be due largely to reduction of venous return and depression of cardiac output in intact animals. In the kidney, ANP increases GFR probably by inducing a relative afferent arteriolar dilation and efferent arteriolar vasoconstriction and an increase in glomerular permeability. The natriuresis is due both to the increase in GFR and to the direct inhibition by ANP of $\mathrm{Na}^{+}$and water reabsorption by inner medullar and cortical collecting duct cells. ANP inhibits secretion of renin, aldosterone, vasopressin and ACTH as well as the stimulation of heart rate mediated by the baroreceptors. Maneuvers that expand plasma volume and increase arterial pressure are associated with increased levels of ANP in plasma. Thus, when blood volume increases stretch may trigger secretion of the peptide and lead to natriuresis and blood pressure reduction. However, the precise role ANP plays in the control of $\mathrm{Na}^{+}$balance, blood volume and blood pressure regulation under normal physiologic conditions is not clear, and there are no known roles for impaired ANP secretion in the pathogenesis or maintenance of essential hypertension. Because of the pharmacologic effects of the natriuretic peptide to induce vasodilatation, hyperfiltration and natriuresis, studies are under way to determine if these peptides may have a therapeutic role in the treatment of hypertension, heart failure, and renal failure. Whether ANP will be a useful therapy for congestive heart failure is uncertain because most of the patients with chronically elevated arterial blood pressures already have increased plasma levels of the peptide.

\section{ENDOTHELIUM-DERIVED RELAXING}

FACTOR: The vascular endothelium produces a labile substance, endotheliumderived relaxing factor (EDRF) that mediates the vasorelaxant actions of various endogenous hormones including acetylcholine. EDRF has been identified as nitric oxide, which is synthesized form the guanidine nitrogen atom of the amino-acid Larginine by the enzyme nitric oxide synthase. Nitric oxide diffuses within the cell or to adjacent cells such as smooth muscle cells, where it stimulates soluble guanylyl cyclase. The resultant increase in cyclic guanosine monophosphate leads to relaxation of the 
vascular smooth muscles and therefore vasodilatation. A number of recent studies in laboratory animals and human subjects have shown that nitric oxide synthesized by the vascular endothelium is an important determinant of resting peripheral vascular resistance and blood pressure. In anesthetized rabbits, inhibition of the nitric oxide synthase activity using substituted arginine analogs acutely increased blood pressure. This hypertensive effect can be reversed by the infusion of arginine. In normal subjects, infusion of L-arginine decreases peripheral vascular resistance, causing hypotension and reflex tachycardia. Because of his apparently important role of nitric oxide in maintaining basal blood pressure it has been proposed that the nitric oxide pathway may be abnormal in patients with hypertension. This view has been supported by recent studies in humans. For example, patients with essential hypertension have a diminished vasoconstrictor response to an infusion of arginine analogs and a reduced arterial vasodilator response to acetylcholine, suggesting that both the basal and stimulated release of nitric oxide is reduced in this disease. In contrast, the response to the endothelium-independent vasodilator nitroprusside was normal in patients with essential hypertension, suggesting that the abnormality is a result of reduced nitric oxide production by the endothelium rather than impaired response in the vascular smooth muscle. The mechanisms responsible for this endothelial dysfunction are not known

\section{ENDOTHELIN:}

In addition to the production of the potent vasodilator nitric oxide, the vascular endothelium produces a potent vasoconstrictor peptide which is called endothelin. At least three endothelin peptides have been identified: endothelin-1, endothelin-2, and endothelin-3. The predominant vascular vasoconstrictor endothelin-1 is formed form proendothelin-1 by the action of a metalloprotease, endothelinconverting enzyme. Endothelin-1 binds to receptors in vascular smooth muscle, which are linked to phospholipids $\mathrm{C}$, resulting in the hydrolysis of phosphoinositide to inositol triphosphate. Increased endothelin activity has been observed in disorders associated with vasoconstriction such as malignant hypertension, heart failure, pulmonary hypertension, contrast-induced acute tubular necrosis, and myocardial infarction. A rare tumor, hemangioendothelioma, can cause hypertension by the secretion of large quantities of endothelin. In addition, the hypertension associated with the use of cyclosporine may be due to increased endothelin production. However, a conclusive role of endothelin in the pathogenesis of essential hypertension has not been demonstrated.

KALLIKREIN-KININ SYSTEM: Kinins are potent vasodilators formed in blood vessels. They are cleaved from the precursor kininogen by the enzymatic action of kallikrein. Kallikrein activity has been noted to be reduced in patients with essential hypertension, suggesting lower vasodilator kinin production. ACE is known to inactivate bradykinin, leading to the suggestion that the hypertensive effect of ACE inhibitors may be due, in part, to increased bradykinin levels. Moreover, the improved insulin sensitivity and glucose utilization observed in diabetic patients treated with ACE inhibitors may be the result of increased kinin levels rather than reduced angiotensin II production.

\section{OTHER HORMNES \& AUTACOIDS:}

Prostaglandins, vasopressin, calcitonin generelated peptide, parathyroid hormone, and parathyroid hormone-related peptide are vasoactive hormones or autacoids that have been implicated in the regulation of blood pressure. Although vasopressin is both a potent vasoconstrictor and prime factor in water reabsorption by the kidney, it does not appear to be a factor in the pathogenesis of essential hypertension. Calcitonin generelated peptide is a potent vasodilator produced in the central nervous system and in autonomic nerves innervating blood vessels. It has been suggested that calcitonin gene- 
related peptide may mediate the hypotensive effect of calcium supplements shown in hypertensive patients. Both infusions of parathyroid hormone and parathyroid hormone-related peptide can produce hypotension. Thus, the hypertension commonly seen in primary hyperparathyroidism is probably due to other factors.

\section{SYMPATHETIC NERVOUS SYSTEM:} Increased activity of the nervous system has been implicated as a contributing factor in the pathogenesis of essential hypertension. This may be due to both genetic and environmental factors. Some patients with hypertension, particularly during the early stages, as well as normotensive offsprings of hypertensive patients have enhanced sympathetic nervous system activity. It has been postulated that impaired baroreceptor function may prevent the normal inhibitory check on increase in sympathetic activity. In addition, the role of stress in the generation and maintenance of hypertension is probably mediated, in part, by activation of the sympathetic nervous system. The mechanisms by which increased sympathetic nervous system activity and catecholamines increase blood pressure is multi-factorial, including augmented vasoconstriction, increased cardiac output, increased activity of the renin-angiotensin system, and enhanced $\mathrm{Na}^{+}$reabsorption by the kidney.

\section{PHEOCHROMOCYTOMA}

Pheochromocytomas are tumors arising from the chromaffin cells of the sympathetic nervous system in the adrenal medulla. They release adrenaline or noradrenaline or both and in some cases dopamine into the circulation, causing hypertension and other signs and symptoms. In addition to catecholamines, pheochromocytomas produce a wide variety of active peptides. These include: vasopressin, somatostatin, substance P, corticotrophin-releasing hormone, adrenocorticotropin, endorphin, lipotropin, vasoactive intestinal polypeptide, interleukin6, parathyroid hormone-related protein, neuropeptide-Y, gastrin, metenkephalin, serotonin, calcitonin, calcitonin-related peptide, neurotensin, pancreastatin, galanin and insulin-like growth factor II. These tumors are found in both sexes at all ages, but usually diagnosed in or after the $4^{\text {th }}$ decade. Global incidence is unknown, but the reported incidence from Sweden is 2 per million. However, it is estimated that 1 in 1000 of patients with diastolic hypertension have pheochromocytoma. Although uncommon, this disorder is important to diagnose because it may prove fatal in pregnant women during delivery and in patients undergoing surgery. Clinical manifestations vary but usually include persistent or paroxysmal hypertension, headache, sweating, tachycardia, tremor, nausea or vomiting, constipation, fatigue, abdominal or chest pain, weight loss and visual disturbances. In few patients the tumor is found incidentally on CT or MRI scan.

Diagnosis and treatment: Assay of catecholamines and their metabolites (metanephrin and vanillymandelic acid) has markedly simplified the diagnosis of this disorder. Glucagon test to induce paroxysm or clonidine test to suppress noradrenaline or therapeutic trial with phenoxybenzamine are rarely needed to establish the diagnosis. Once the diagnosis is confirmed, the tumor must be localized to facilitate its surgical removal. CT and MRI are very efficient for that purpose. Radio-isotope scanning using iodine is useful for very small tumors and for extra-abdominal catecholamine-producing tumors (MIBG). Surgical treatment is curative, but preoperative treatment to control hypertension is mandatory. Drugs used for such therapy are adrenergic antagonists and include phentolamine, phenoxy benzamine, and prazosin. Propranolol (a beta blocker) is used to control marked tachycardia and arrhythmia, but it should not be given until alpha receptor blockade is established. 


\section{REFRENCES}

1. Don BR, Schambelan M. Endocrine hypertension. In: Basic \& Clinical Endocrinology, $6^{\text {th }}$ edition. Greenspan FS, Gardner DG (editors). McGraw- Hill 2001.

2. Krieger CE, Dzau VJ. Molecular biology of hypertension. Hypertension 1991; 18(suppl): 13-15.

3. Lifton RP. Genetic determinants of human hypertension. Proc Soc Natl Acad Sci 1995; 92: 14951499.

4. Lifton RP, Mann SJ. A chimaeric 11 bhydroxylase/aldosterone synthase gene causes glucocorticoid-remediable aldosteronism and human hypertension. Nature 1992; 355: 262.

5. Lowenstein CJ, Pickering TG. Nitric oxide: A physiologic messenger. Ann Intern Med 1994; 120: 227-230.

6. Gordon RD. Mineralocorticoid hypertension. Lancet 1994; 344: 240-241.

7. Genest J, Cantin M. The atrial natriuretic factor: its physiology \& biochemistry. Rev Physiol Biochem Pharmacol 1988; 110: 1-6.

8. Irony I, Kater CE. Correctable subsets of primary hyperaldosteronism: Primary adrenal hyperplasia and renin responsive adenoma. Am J Hypertension 1990; 3: 576-580.

9. Anderson GH, Williams JR, Cohen F, et al. The effect of age on prevalence of secondary forms of hypertension in 4429 consecutively referred patients. J Hypertension 1994; 12: 600-610.

10. Baxter JD. The endocrinology of hypertension. In: Endocrinology \& Metabolism, $3^{\text {rd }}$ edition. Felig $P$, Baxter JD, Frohman LA (editors). McGraw-Hill, 1995.

11. Biglieri EG, Kater CE. 17 $\alpha$-hydroxylation deficiency. Endocrinol Metab Clin North Am 1991; 20: 257-260.

12. Funder JW. Mineralocorticoid action: Target tissue specificity in enzyme, not receptor, mediated. Science 1988; 110: 1-5.
13. Chen SY, Davidson RA. Epithelial sodium channel regulated by aldosterone-induced protein SGK. Proc Soc Natl Acad Sci 1999; 96: 2514-2518.

14. Kater CE, Gordon RD. Stimulation and suppression of the mineralocorticoid hormones in normal subjects and adrenocortical disorders. Endocrinol Rev 1989; 11: 149-151.

15. Reaven GM. Pathophysiology of insulin resistance in human disease. Physiol Rev 1995; 75: 473-476.

16. Schamberlan M. Liquorice ingestion and blood pressure regulating hormones. Steroids 1994; 59: 127132.

17. Shiffrin EL. Endothelin: Potential role in hypertension and vascular hypertrophy. Hypertension 1995; 25: 1135-1139.

18. Shimkets RA. Liddle syndrome: heritable human hypertension caused by mutation in the $\beta$ subunit of the epithelial sodium channel. Cell 1994; 79: 407-410.

19. Ulick S. Two uncommon causes of mineralocorticoid excess: Syndrome of apparent mineralocorticoid excess and glucocorticoidremediable aldosteronism. Endocrinol Metab North Am 1991; 20: 269-272.

20. Wilson RC, Morley D. Several homozygous mutations in the gene for $11 \beta$-hydroxysteroid dehydrogenase type 2 in patients with apparent mineralocorticoid excess. J Clin Endocrinol Metab 1995; 80: 3145-3147.

21. White PC. Disorders of aldosterone biosynthesis and action. N Eng J Med 1994; 331: 250-255.

22. Williams GH. Guardian of the gate: Receptors, enzymes and mineralocorticoid function. J Clin Encocrinol Metab 1991; 74: 961-963.

23. Peaston RT, Lennard TW, Lai LC. Overnight excretion of urinary catecholamines and metabolites in the detection of pheochromocytoma. J Clin Endocrinol Metab 1996; 81: 1379-1382.

24. Werbel SS, Ober KP. Pheochromocytoma: update on diagnosis, localization and management. Med Clin North Am 1995; 79: 131-141. 